\title{
Spectroscopic Observation of He Arcjet Plasma Expanding through a Converging and Diverging Slit Nozzle
}

\author{
Kazuki Kozue ${ }^{1}$, Shinichi Namba ${ }^{1, *}$, Takuma Endo ${ }^{1}$, Ken Takiyama $^{1}$, and Naoki Tamura ${ }^{2}$ \\ 1 Graduate school of Engineering, Hiroshima University, 1-4-1 Higashi-Hiroshima, Hiroshima 739-8527, \\ Japan \\ 2 National Institute for Fusion Science, 322-6 Oroshi-cyo, Toki, Gifu 509-5292, Japan \\ *Email: namba@hiroshima-u.ac.jp
}

\begin{abstract}
An arcjet plasma generator with a converging and diverging slit nozzle was constructed. This plasma source allowed us to directly observe the arc plasma in the discharge section, which provided useful information about a transition from ionizing thermal plasma to recombining phase. Spatial distributions of the electron temperature and density in the rectangular shaped anode nozzle were evaluated by visible emission spectroscopy. The temperature and density for a discharge current of $20 \mathrm{~A}$ were determined to be $\sim 0.18 \mathrm{eV}$ and $\sim 3.7 \times 10^{13} \mathrm{~cm}^{-3}$, respectively, at the nozzle throat. These values were compared with those calculated by the gas dynamic theory based on one dimensional slit nozzle.
\end{abstract}

Keywords: Arcjet plasma, slit nozzle, plasma spectroscopy.

ENGINEERING JOURNAL Volume 17 Issue 5

Received 9 April 2013

Accepted 24 July 2013

Published 31 December 2013

Online at http://www.engj.org/

DOI:10.4186/ej.2013.17.5.7

This paper is based on the oral presentation at the 1st Joint Conference on "Renewable Energy and Nanotechnology" (JCREN 2012) in Bangkok, Thailand, 19-20 November 2012. 


\section{Introduction}

Arcjet plasmas have been applied to various scientific and engineering fields [1], such as metal processings (welding and cutting) [2], chemical vapor deposition (CVD) [3], waste treatment [4] and plasma propulsion $[5,6]$. We have developed various arcjet plasma sources with supersonic conical nozzles so far [7]. However, the arcjet generators have axisymmetric structures, so that the arc discharging section cannot be observed directly. The temperature, density and flow of the arc plasmas, therefore, have not been well understood inside the anode nozzle. The observation of the plasma production volume could provide useful information for long term operation of the arc discharge [8]. The rectangular shaped nozzle with a converging and diverging section was constructed by Ogiwara et al. [9]. They revealed that the transition from low voltage mode to high voltage one occurred and the latter mode showed the favorable condition for high thrust power. However, the details of the characteristics of the plasma parameters and transition from an ionizing to recombining plasma were not investigated.

We have also developed the arcjet plasma device with a slit shaped anode nozzle. Although we successfully obtained the variations of the plasma temperature and density on the expansion axis by emission spectroscopy [8], their two-dimensional (2D) distributions have not been observed so far, which are very important to clarify the characteristics of plasmas and expansion dynamics. To this end, the spectroscopic observation was carried out by using a high resolution spectrometer, and 2D spatial distributions of the temperature and density inside the nozzle were obtained by analyzing continuum and line emission spectra on $\mathrm{He}$ arc discharges. Consequently, the temperature and density were evaluated to be $\sim 0.18 \mathrm{eV}$ and $\sim 3.7 \times 10^{13} \mathrm{~cm}^{-3}$, respectively, for a $20 \mathrm{~A}$ He discharge at the nozzle throat $(x=0 \mathrm{~mm})$. The experimental values obtained were compared with those calculated by the gas dynamic theory for 1D slit nozzle. The methods to determine the plasma parameters and a comparison between the experimental and analytical values are described in detail.

\section{Experimental Setup}

We constructed an arcjet plasma generator that had a rectangular converging and diverging slit nozzle, allowing to directly observe the thermal arc plasma inside the nozzle. Figure 1(a) and (b) show a photograph and schematic diagram of the arcjet plasma generator, respectively. A pair of anode made of molybdenum served as the converging and diverging slit nozzle, and the separation between them was set to $0.5 \mathrm{~mm}$. A cathode was needle-shaped CeW $\operatorname{rod}(2.4 \mathrm{~mm} \phi)$. The discharge current, voltage and operation gas are $20 \mathrm{~A}, 30 \mathrm{~V}$ and helium, respectively. In order to examine the characteristics of the expanding arc plasma inside the nozzle, a visible spectrometer with a focal length of $0.5 \mathrm{~m}$ was used. The diffraction gratings were 1200 and 3600 grooves $/ \mathrm{mm}$. The detector was a charged coupled device (CCD) camera. The emission was imaged by a lens onto an end of optical bundled fiber with 48 cores. The light through the fiber was imaged by a lens again onto an entrance slit of the spectrometer. The 2D spatial distribution of the plasma emission was obtained by moving the focus position using an optical stage. Spectral sensitivity was calibrated by using tungsten ribbon and xenon discharge lamps.

(a)

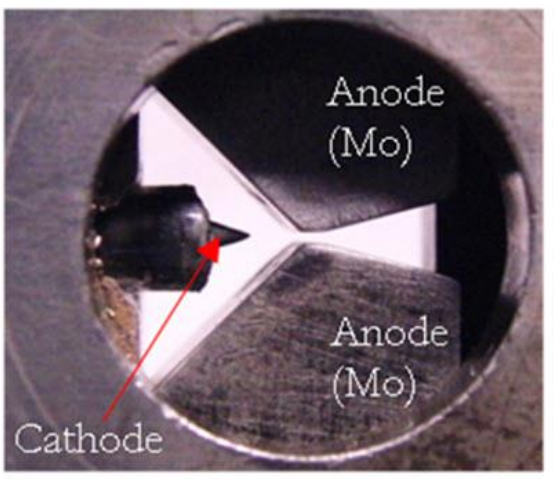

(b)

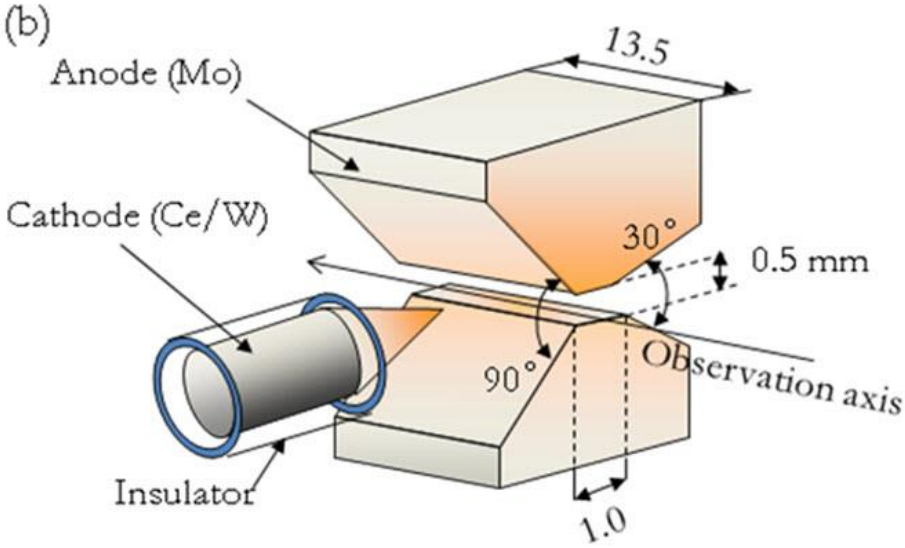

Fig. 1. Photograph (a) and schematic diagram (b) of the arcjet plasma generator. 


\section{Results and Discussion}

\subsection{Emission Spectra of He Arcjet Plasma}

Figure 2 shows the typical emission spectra observed for the helium arcjet plasma. The intense continuum spectra as well as line spectra of neutral helium were observed. In this study, from the continuum spectra (recombination radiation) and the line spectra (Boltzmann plot) the electron temperature was determined, while from the line shape (stark broadening spectra) the electron density was evaluated.

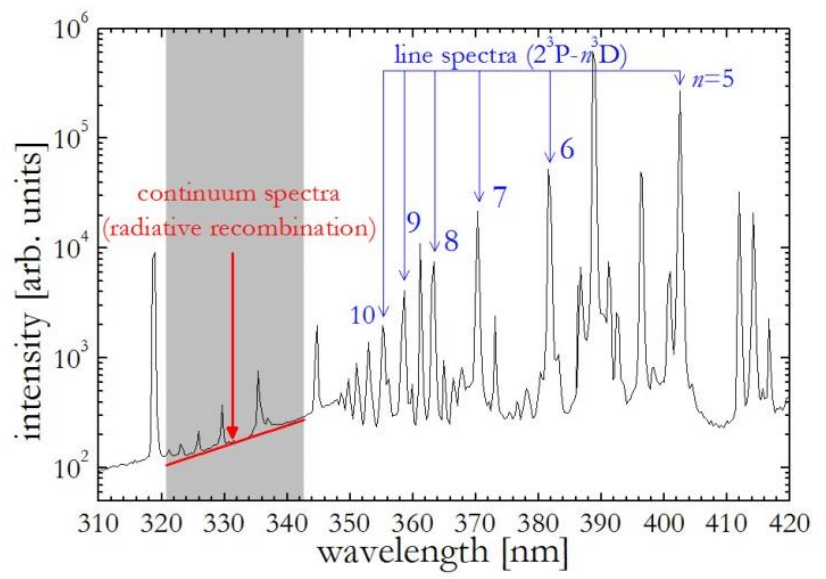

Fig. 2. Typical emission spectra at a He discharge current of $20 \mathrm{~A}$ and its voltage of $\sim 30 \mathrm{~V}$.

\section{2. $\quad$ Electron Temperature}

\subsubsection{Recombination Radiation}

The continuum spectra due to the radiative recombination were observed, by which the electron temperature can be evaluated. The emissivity of the spectra $\varepsilon(v)$ is expressed by [10],

$$
\varepsilon(v)=\frac{b^{7}}{8 \pi^{\frac{9}{2}} m_{e}^{3} e^{6}} \cdot \frac{v^{3}}{c^{2}} \cdot \frac{g}{g_{z}} \sigma(v) n_{e} n_{z}\left(\frac{E_{H}}{k_{B} T_{e}}\right)^{\frac{3}{2}} \exp \left[\frac{1}{k_{B} T_{e}}(\chi-b v)\right]
$$

where $n_{e}$ is the electron density, $n_{z}$ is the density of ions, $T_{e}$ is the electron temperature, $g$ and $g_{z}$ are the statistical weights of the ground state of the atom and $\mathrm{He}^{+}$ion, respectively, $\sigma$ is the photoionization cross section for $\mathrm{He}\left(1 \mathrm{~s}^{2}\right), E_{H}$ is the ionization energy of the $\mathrm{H}$ atom, $\chi$ is the ionization energy from $\mathrm{He}\left(1 \mathrm{~s}^{2}\right)$, and the other notations have the usual meanings. The temperature is obtained from the tangent of a straight line of $\log \left[\varepsilon(v) /\left(\sigma(v) v^{6}\right)\right]$ plotted against photon energy $h v$. In this study, the transition of He $2^{3} \mathrm{P}-n^{3} \mathrm{D}$ series was used (see the gray region in Fig. 1).

\subsubsection{Boltzmann Plot}

Among high Rydberg states the condition of local thermal equilibrium (LTE) is fulfilled, so that the population density $n(i)$ of the $i$ th state is described by the Boltzmann relation $[10,11]$ :

$$
\frac{n(p)}{n(q)}=\frac{g(p)}{g(q)} \exp \left[-\frac{E_{p}-E_{q}}{k_{B} T_{e}}\right]
$$

where $g(i)$ is the statistical weight of the $i$ th level and $E(\imath)$ is its excitation energy. By observing line spectra of He I $2{ }^{3} P-n{ }^{3} D$ up to $n=10$, the population densities can be derived. From the slope of $\log (n / g)$ against the excitation energy, the electron temperature is evaluated from the Boltzmann equation. 


\subsection{Electron Density}

The line spectra in plasmas are broadened due to various effects. In high-density plasmas, the Stark broadening dominates over the other effects (resonance and van der Waals ones), and the line width is a function of electron temperature and density [11]. Here, the line spectra observed can be expressed by a Voigt function, which is the convolution of a Gaussian (Doppler and instrumental width) and Lorentzian (Stark broadenings) shapes. The Stark width (full width at half maximum: FWHM), $\Delta \lambda_{\mathrm{s}}$, for nonhydrogenic atoms is approximately given by the following equation $[10,11]$ :

$$
\Delta \lambda \approx 2 \omega\left[1+1.75 \times 10^{-4} n_{e}^{\frac{1}{4}} \alpha\left(1-0.068 n_{e}{ }^{\frac{1}{6}} T_{e}^{\frac{1}{2}}\right)\right] \times 10^{-16} n_{e}
$$

Here, $n_{\mathrm{e}}$ is the electron density $\left(\mathrm{cm}^{-3}\right)$ and $T_{\mathrm{e}}$ is the electron temperature $(\mathrm{K}), \omega$ is the half width at half maximum (HWHM) due to electron impacts and $\alpha$ is the ion broadening parameter. Since these variables depend on the temperature, we inferred them for various temperatures by using the values tabulated in Ref. 11. In this study, the Stark FWHM was extracted by fitting the experimental data with the Voigt function. Here, the line spectrum of He I $438.8 \mathrm{~nm}\left(2^{1} \mathrm{P}-5^{1} \mathrm{D}\right)$ was used, and the instrumental and Doppler $\left(T_{\mathrm{g}} \sim 0.16\right.$ $\mathrm{eV}$ ) width were 18.6 and $6.7 \mathrm{pm}$, respectively.

\subsection{D Distributions of Plasma Temperature and Density}

Figure 3(a), (b) and (c) show 2D spatial distributions of the emission intensity $\left(382.0 \mathrm{~nm}\left(2^{3} \mathrm{P}-6^{3} \mathrm{D}\right)\right.$ ), electron temperature and density, respectively. The highest temperature and density at a discharge current of $20 \mathrm{~A}$ were evaluated $\sim 0.18 \mathrm{eV}$ and $3.7 \times 10^{13} \mathrm{~cm}^{-3}$ at the nozzle throat, respectively. It was found that the emission intensity and the electron density gradually decreased downstream from the throat. However, distinct decrease of the temperature was not observed. The reason for this can be interpreted by that we evaluated the temperature by analyzing the emission spectra integrated over line-of-sight. This implies that the determination of the local temperature requires the spatial distribution along the slit depth direction.
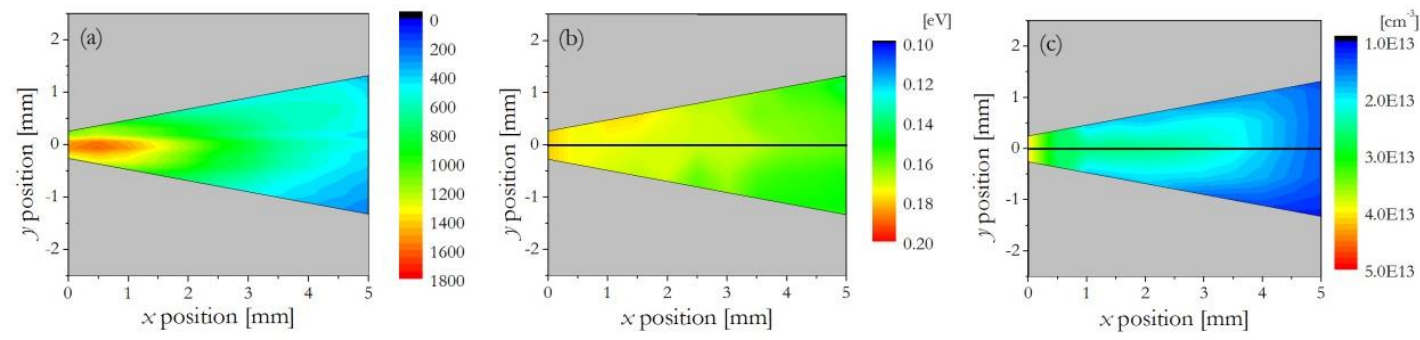

Fig. 3. 2D spatial distributions of the emission intensity (a), electron temperature (b) and density (c).

Figure 4 shows the comparisons of the electron temperature (a) and density (b) with the analytical curves along the expansion axis $(y=0 \mathrm{~mm})$. Here, the calculated curve is based on the gas dynamics theory for the expansion through quasi $1 \mathrm{D}$ nozzle [12]. The temperature $T$ and density $n$ in terms of the Mach number $M$ are given by,

$$
T=T^{*}\left(1+\frac{\gamma-1}{2} M^{2}\right)^{-1}, \quad n=n^{*}\left(1+\frac{\gamma-1}{2} M^{2}\right)^{\frac{-1}{1-\gamma}}
$$

where $\gamma$ is the specific heat ratio. $T^{*}$ and $n^{*}$ represent the values at the throat $(M=1)$ and are set to be 0.18 $\mathrm{eV}$ and $3.7 \times 10^{13} \mathrm{~cm}^{-3}$, respectively. The temperature and density obtained experimentally, however, are not in agreement with the theoretical curve. Shown in Fig. 5 is the reason for this disagreement. The arc plasma expands from the cathode tip as shown in Fig. 5(a). On the other hand, the gas flow is considered to be constant along horizontal direction (see Fig. 5(b)). Thus, the electron temperature and density distribution could not agree with the values calculated by the simple $1 \mathrm{D}$ gas dynamics theory. 

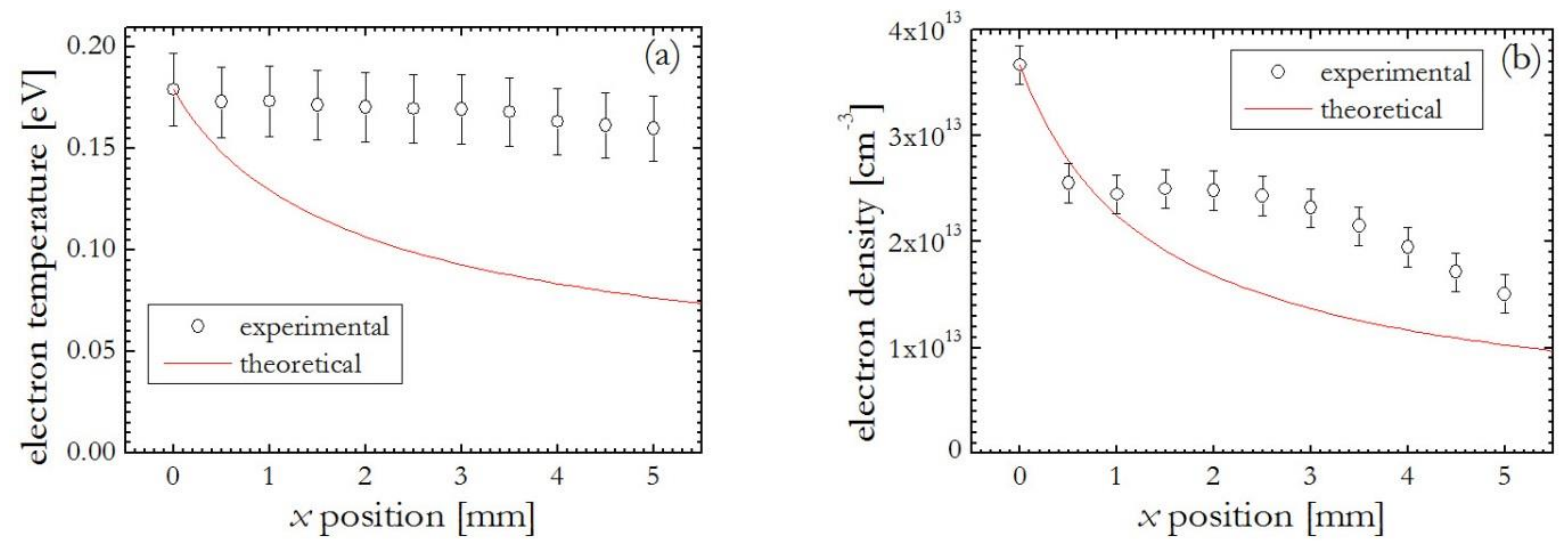

Fig. 4. Comparison of the electron temperature (a) and density (b) with their theoretical curves.

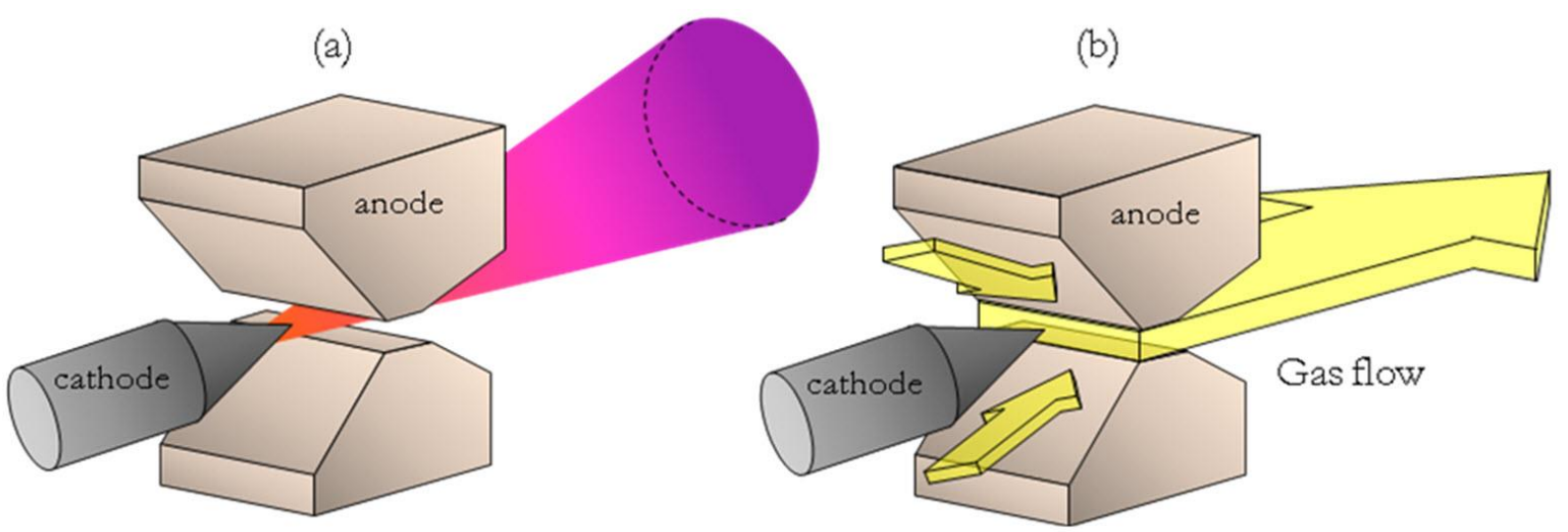

Fig. 5. The difference of expansion flows from the slit nozzle for arc jet plasma (a) and He neutral gas (b).

\section{Conclusion}

In order to obtain the $2 \mathrm{D}$ spatial distributions of the electron temperature and density in the arc discharge section, the measurement by using visible emission spectroscopy were made. The plasma temperature and density for He plasma were evaluated to be $0.18 \mathrm{eV}$ and $3.7 \times 10^{13} \mathrm{~cm}^{-3}$ at the nozzle throat, respectively, by the continuum and line spectra. The electron density gradually decreased downstream from the throat, whereas no variation of the temperature was observed. The reason for this could be explained by that the parameters were derived by the integrated spectra over line-of-sight. The experimental result was compared with the values calculated by the conventional gas dynamics theory. However, they were not in agreement, since the plasma expanded from cathode tip to vacuum through the slit nozzle, whereas the neutral He gas did with the constant flow along the slit nozzle.

\section{References}

[1] M. I. Boulos, P. Fauchais, and E. Pfender, Thermal Plasmas: Fundamentals and Applications. Plenum Press, 1994.

[2] K. Nishiguchi, T. Ohji, M. Yoshida, K. Kuwata, W. Shimada, S. Hiromoto, and M. Moriyasu, Japan Welding Society, vol. 43, pp. 138-139, 1988.

[3] D. M. Dobkin and M. K. Zuraw, Principles of Chemical Vapor Deposition. Kluwer Academic, 2003.

[4] T. Amemiya, T. Kikuchi, and Y. Gohda, "Treatment of incineration ash and flyash by melting process utilizing plasma," Journal of Plasma and Fusion Research, vol. 73, no. 9, pp. 928-934, 1997.

[5] J. D. Anderson, Jr., Modern Compressible Flow with Historical Perspective, 2nd ed. New York: McGraw Hill, 1989.

[6] G. P. Sutton, Rocket Propulsion Elements: An Introduction to the Engineering of Rockets. John Wiley \& Sons, 
1992.

[7] S. Namba, K. Nakamura, N. Yashio, S. Furukawa, K. Takiyama, and K. Sato, "Transition from thermal to recombining plasma in a free expanding arc jet plasma generator," Journal of Plasma and Fusion Research SERIES, vol. 8, pp. 1348-1352, 2009.

[8] S. Namba, N. Yashio, K. Kozue, K.Nakamura, T. Endo, K. Takiyama, and K. Sato, "Spatially resolved spectroscopic study of arcjet helium plasma expanding through a rectangular converging and diverging nozzle," Jpn. J. Appl. Phys., vol. 48, no. 11R, p. 116005, 2009.

[9] K. Ogiwara, S. Hosoda, I. Kimura, H. Kuninaka, K. Toki, and K. Kuriki, "The observation of discharge phenomena in low power DC arcjets," Journal of the Japan Society for Aeronautical and Space Sciences, vol. 48, no. 559, pp. 250-256, 2000 (in Japanese).

[10] R. H. Huddlestone and S. L. Leonard, Plasma Diagnostic Techniques. New York: Academic, 1965.

[11] H. R. Griem, Spectral Line Broadening by Plasmas. New York: Academic, 1974.

[12] M. D. Morse, "Supersonic beam sources," in Atomic, Molecular, and Optical Physics: Atoms and Molecules, vol. 29B, F. B. Dunning and R. G. Hulet, Eds. San Diego,CA: Academic, 1996. 